\title{
MINIMUM-WEIGHT TWO-CONNECTED SPANNING NETWORKS
}

\author{
Clyde L. MONMA \\ Bell Communications Research, Morristown, NJ 07960, USA
}

\section{Beth Spellman MUNSON}

AT\&T Bell Laboratories, Holmdel, NI 07733, USA

\author{
William R. PULLEYBLANK \\ University of Waterloo, Waterloo, Ont., Canada N2L 3G1
}

Received 9 May 1986

Revised manuscript received 8 August 1988

\begin{abstract}
We consider the problem of constructing a minimum-weight, two-connected network spanning all the points in a set $V$. We assume a symmetric, nonnegative distance function $d(\cdot)$ defined on $V \times V$ which satisfies the triangle inequality. We obtain a structural characterization of optimal solutions. Specifically, there exists an optimal two-connected solution whose vertices all have degree 2 or 3 , and such that the removal of any edge or pair of edges leaves a bridge in the resulting connected components. These are the strongest possible conditions on the structure of an optimal solution since we also show that any two-connected graph satisfying these conditions is the unique optimal solution for a particular choice of 'canonical' distances satisfying the triangle inequality. We use these properties to show that the weight of an optimal traveling salesman cycle is at most $\frac{4}{3}$ times the weight of an optimal two-connected solution; examples are provided which approach this bound arbitrarily closely. In addition, we obtain similar results for the variation of this problem where the network need only span a prespecified subset of the points.
\end{abstract}

Key words: Spanning networks, two-connectivity, traveling salesman problem.

\section{Introduction}

Consider a set of vertices $V$ with a nonnegative, symmetric distance function (or metric) $d(\cdot)$ defined on $V \times V$ which satisfies the triangle inequality, i.e.,

(a) $d(u, u)=0$,

(b) $d(u, v) \geqslant 0$,

(c) $d(u, v)=d(v, u)$,

(d) $d(u, w) \leqslant d(u, v)+d(v, w)$,

for all $u, v$ and $w$ in $V$. We call $d(u, v)$ the weight or length of the edge $(u, v)$. A subset of edges $E \subseteq V \times V$ defines a network or graph $G=(V, E)$ whose weight is given by $d(E)=\sum_{(u, v) \in E} d(u, v)$. In order to avoid trivial special cases, we assume throughout that $|V| \geqslant 3$. We consider the problem of constructing a minimum-weight two-connected network spanning the vertices in $V$. 
The widely-studied traveling salesman problem $[1,5,24,30]$ is closely-related in that the objective is to find a minimum-weight (Hamiltonian) cycle spanning all vertices in $V$. In fact, it is easy to see [14] that the problem of determining if a graph $G=(V, E)$ contains a Hamiltonian circuit can be reduced to the minimum-weight two-connected spanning network problem. (Assign a weight of one to the edges in $E$ and a weight of two to all nonedges; then there is a Hamiltonian cycle in $G$ if and only if an optimal solution has weight $|V|$.) Hence, the problem is NP-complete. (See [18] for an overview of the theory of computational complexity.)

The primary motivation for studying the minimum-weight two-connected spanning network problem is because of its application to the design of survivable communication and transportation networks. The simplest network design problem involves finding a minimum-weight spanning tree and is easily solved [20] for arbitrary weights. An important survivability consideration is for a constructed network to remain connected even after the removal of some edges or vertices. These considerations have been explored from several points of view $[6,34]$ building on previous work on the traveling salesman problem.

In Section 2 we obtain a number of structural properties of optimal two-connected spanning networks. In particular, we show that there exists an optimal two-connected solution whose vertices all have degree 2 or 3 , and such that the removal of any edge or pair of edges leaves a bridge in the resulting connected components. This is the strongest possible restriction on the structure of an optimal solution since we also show that any two-connected graph satisfying these conditions is the unique optimal solution for a particular choice of 'canonical' distance function. These conditions may be useful in restricting the search for an optimal solution.

In Section 3 we use these structural properties to give a simple proof that the weight of an optimal traveling salesman cycle is no greater than $\frac{4}{3}$ times the weight of an optimal two-connected solution; examples are provided which approach this bound arbitrarily closely. This result was conjectured and almost proved by Frederickson and Ja'Ja' [15]. We discuss the relationship to their work in Section 3. We also include a result due to W.H. Cunningham which relates the weight of an optimal two-connected solution to the value of a well-known linear programming relaxation of the traveling salesman problem. We extend some of our results to the Steiner version of the minimum-weight two-connected network problem where only a prespecified subset of the vertices must be spanned. Concluding remarks and open problems are presented in Section 4.

\section{Structural properties}

In this section we develop strong properties describing the structure of optimal two-connected spanning networks. We first give some useful definitions and lemmas.

A cut vertex (resp. bridge) in a graph $G=(V, E)$ is a vertex (resp. edge) whose removal increases the number of connected components in $G$. A connected graph 
$G=(V, E)$ is called two-vertex (resp. two-edge) connected if ${ }^{\circ}$ contains no cut vertex (resp. bridge). Clearly, two-vertex connected implies two-edge connected, although the converse is not true.

Lemma 1 [2]. The following are equivalent statements for a graph $G=(V, E)$.

(a) $G$ is two-vertex connected.

(b) Every two vertices of $G$ lie on a common cycle.

(c) Every two edges of $G$ lie on a common cycle.

(d) Every vertex and edge of $G$ lie on a common cycle.

These equivalences holds for two-edge connected graphs by replacing 'cycle' by 'circuit', i.e., allowing vertices, but not edges, to be repeated.

Proof. Easy.

We consider the problem of constructing a minimum-weight two-vertex connected network spanning all vertices in $V$ under the assumption that the weight function satisfies the triangle inequality. Under this assumption it is easy to show that the minimum weight of a two-edge connected spanning network is equal to the minimum weight of a two-vertex connected spanning network [15, Section 3, first paragraph]. As a result, we will often use the term 'two-connected' later in the paper without specifying 'edge' or 'vertex'.

An approach which is used repeatedly later is to make a local change to an optimal solution which preserves feasibility and optimality. The following lemma shows that a wide class of local changes preserves two-connectedness.

Lemma 2. Let $G=(V, E)$ be a two-connected graph with $G^{\prime}=\left(V^{\prime}, E^{\prime}\right)$ a subgraph of $G$ induced by $V^{\prime}$. Then replacing $E^{\prime}$ in $G$ by any collection of edges $E^{\prime \prime}$ defined on $V^{\prime}$, where $G^{\prime \prime}=\left(V^{\prime}, E^{\prime \prime}\right)$ is two-connected, results in a graph $G^{*}=\left(V,\left(E \backslash E^{\prime}\right) \cup E^{\prime \prime}\right)$ which is two-connected.

Proof. Suppose $G^{*}$ is not two-connected and let $v$ be a cut vertex. Since $G$ is two-connected, every component of $G^{*}(V \backslash\{v\})$ must contain a vertex of $V^{\prime}$. Since $G^{\prime}$ is two-connected, we can find a path in $G^{*}$ which avoids $v$ between these vertices in any two of these components. This contradicts $v$ being a cut vertex.

Theorem 3. For any set of vertices $V$ with distance function $d(\cdot)$ on $V \times V$, there exists a minimum-weight two-connected graph $G=(V, E)$ satisfying the following conditions.

(a) Every vertex in $G$ has degree 2 or 3.

(b) Deleting any edge or pair of edges in $G$ leaves a bridge in one of the resulting connected components of $G$.

Proof. Let $G=(V, E)$ be a minimum-weight two-vertex connected solution not satisfying condition (a). There must be a vertex $u$ with distinct neighbors $a, b, c$ 
and $d$. Since $G$ is two-vertex connected, there must be paths between any pair of these four neighbors which avoid vertex $u$.

Case $A$. If one of these paths, say from vertex $a$ to vertex $c$ includes one of the other vertices, say $b$, then by Lemma 2 we may delete the edge $(u, b)$ and still remain two-vertex connected. (See Fig. 1(a).)

Case $B$. If no path as described in Case A exists between any pair of the vertices $a, b, c$ and $d$, then the paths from $a$ to $b, a$ to $c$, and $a$ to $d$ are of the form shown in Fig. 1(b) (with possibly the identities of vertices $b, c$ and $d$ interchanged). We consider here the case where vertices $v$ and $w$ are distinct. Replacing the edges $(u, b)$ and $(u, c)$ by the edge $(b, c)$ preserves two-connectedness, by Lemma 2 , and does not increase the cost by the triangle inequality.

Case $C$. Consider the case shown in Fig. 1(c) where $v=w$. Let $a_{1}, b_{1}, c_{1}$, and $d_{1}$ be the last vertices in paths from $u$ to $v$ through $a, b, c$ and $d$, respectively, which need not be distinct from $a, b, c$, and $d$. Replacing the edges $(u, a)$ and $(u, b)$ by the edge $(a, b)$, and replacing the edges $\left(v, b_{1}\right)$ and $\left(v, c_{1}\right)$ by the edge $\left(b_{1}, c_{1}\right)$ preserves two-connectedness, by Lemma 2 , and does not increase the cost by the triangle inequality.
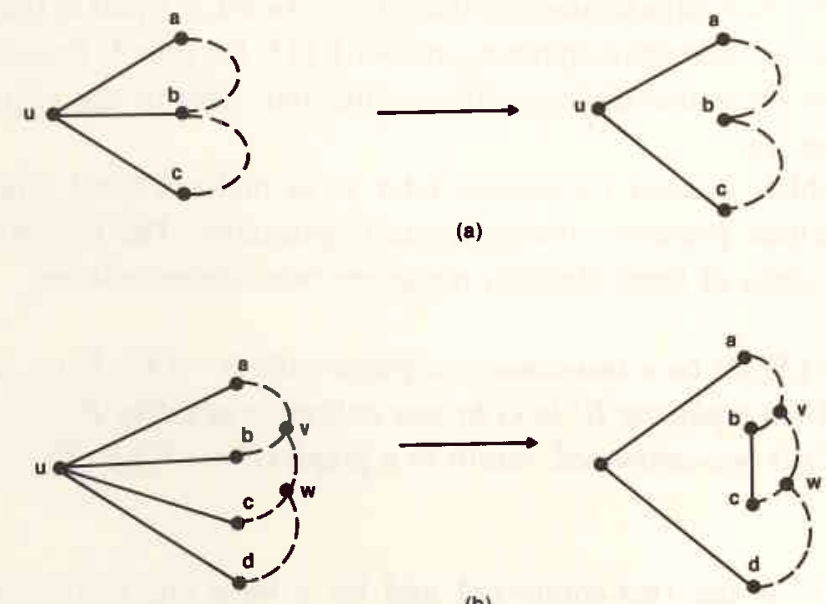

(b)
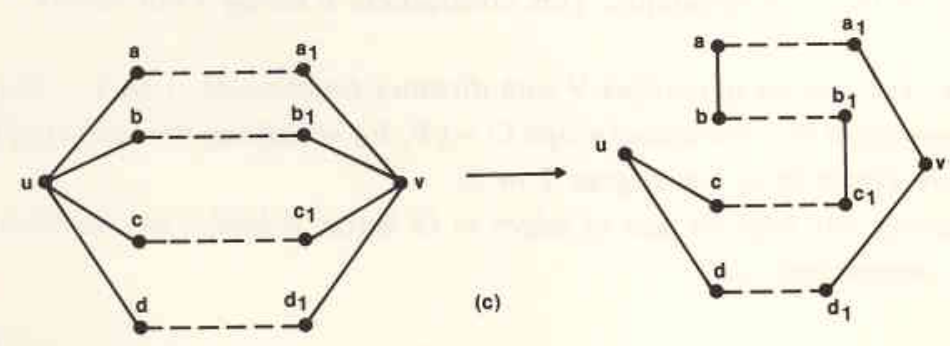

Fig. 1. Cases for Theorem 3, condition (a). 
In all three cases, the degree of vertex $u$ is reduced by at least one, the degree of all other vertices remain the same, and the solution is still two-vertex connected and optimal. Repeating this process establishes condition (a).

Let $G=(V, E)$ be a minimum-weight two-connected solution satisfying condition (a) but not condition (b). Suppose there exists an edge which can be deleted without creating a bridge. (See Fig. 2(a).) Doing so results in a two-edge connected solution whose cost is no greater. A cut-vertex in a two-edge connected graph must have degree at least four, so in fact this solution must also be two-vertex connected. Hence we can simply delete all such edges, and so assume that $G$ is edge minimal.

Suppose there exist edges $(u, v)$ and $(w, x)$, with possibly $x=v$, whose deletion results in no bridge, i.e., the connected components are all two-edge connected. Again, since (a) holds, each component is two-vertex connected.

Case $A$. The edges are of the form $(u, v)$ and $(v, w)$ with $u \neq w$. Since $G$ is two-vertex connected, $u$ and $w$ must be in the same connected component, which we have seen is two-vertex connected. Hence, by Lemma 1, there is a vertex-induced subgraph with $(u, v)$ and $(v, w)$ as a 'two-chord' of a cycle, as shown in Fig. 2(b). Since $G$ is edge minimal two-connected, we may assume that $a, b, c$ and $d$ are

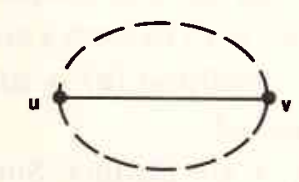

(a)

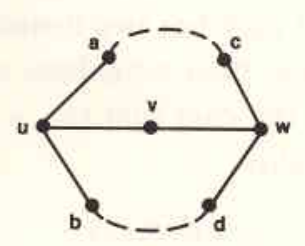

(b)

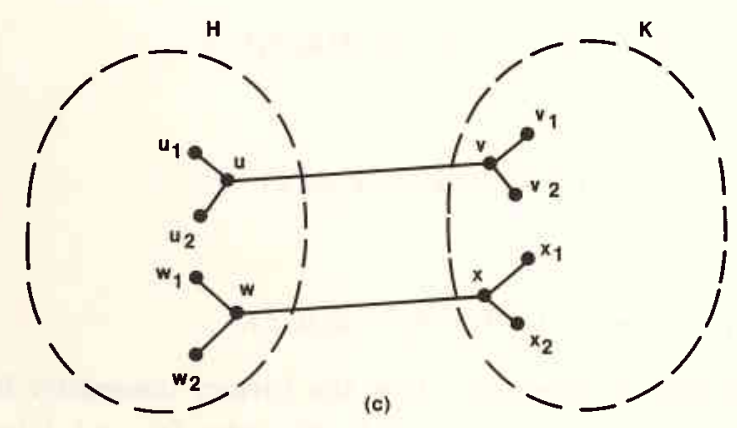

Fig. 2. Cases for Theorem 3, condition (b). 
different from $u$ and $w$, although it may be the case that $a=c$ and/or $b=d$. Now by the triangle inequality,

$$
\begin{aligned}
& d(a, v) \leqslant d(a, u)+d(u, v), \\
& d(b, v) \leqslant d(b, u)+d(u, v), \\
& d(c, v) \leqslant d(c, w)+d(w, v), \\
& d(d, v) \leqslant d(d, w)+d(w, v) .
\end{aligned}
$$

Therefore, at least one of the following must hold:

$$
\begin{aligned}
& d(a, v) \leqslant d(a, u)+d(v, w), \\
& d(b, v) \leqslant d(b, u)+d(v, w), \\
& d(c, v) \leqslant d(c, w)+d(u, v), \\
& d(d, v) \leqslant d(d, w)+d(u, v) .
\end{aligned}
$$

Without loss of generality, assume that the first inequality holds. We now replace the edges $(a, u)$ and $(v, w)$ by the edge $(a, v)$ to form a cycle in the induced subgraph. Clearly, the cost does not increase, condition (a) is still satisfied, and, by Lemma 3 , the graph is still two-vertex connected.

Case $B$. The four vertices $u, v, w, x$ are distinct. Since $G$ is two-connected and edge-minimal we may assume that the removal of $(u, v)$ and $(w, x)$ yields exactly two two-vertex connected components with $u$ and $w$ in one component $H$, and with $v$ and $x$ in component $K$. Also, by two-connectivity and condition (a), these four vertices are all of degree three and each has two distinct neighbors as shown in Fig. 2 (c). Also, since $G$ is edge-minimal, their neighbors are different from $u, v, w$ and $x$, as shown. It may, however, be the case that $\left\{u_{1}, u_{2}\right\} \cap\left\{w_{1}, w_{2}\right\} \neq \emptyset$, or $\left\{v_{1}, v_{2}\right\} \cap$ $\left\{x_{1}, x_{2}\right\} \neq \emptyset$. By the triangle inequality,

$$
d\left(u_{1}, v_{1}\right) \leqslant d\left(u_{1}, u\right)+d(u, v)+d\left(v, v_{1}\right)
$$

and

$$
d\left(w_{1}, x_{1}\right) \leqslant d\left(w_{1}, w\right)+d(w, x)+d\left(x, x_{1}\right) .
$$

Therefore, either

$$
d\left(u_{1}, v_{1}\right) \leqslant d\left(u_{1}, u\right)+d\left(v, v_{1}\right)+d(w, x)
$$

or

$$
d\left(w_{1}, x_{1}\right) \leqslant d\left(w_{1}, w\right)+d\left(x, x_{1}\right)+d(u, v) .
$$

Suppose, without loss of generality, that the former inequality holds. We replace the edges $\left(u_{1}, u\right),\left(v, v_{1}\right)$ and $(w, x)$ in $G$ by the edge $\left(u_{1}, v_{1}\right)$. Clearly the cost does not increase, and condition (a) still holds. 
It remains to be shown that the new graph is two-vertex connected. By Lemma 1 , it suffices to show that any pair of vertices $a$ and $b$ lie on a common cycle. Suppose first that both $a$ and $b$ are in the two-vertex connected component $H$. (The proof is similar if both are in $K$.) If the cycle in $G$ did not contain the edge $\left(u_{1}, u\right)$ then we are done. If it did, then we replace the edge $\left(u_{1}, u\right)$ in the cycle by a path through $K$ from $u_{1}$ to $u$ using the edges $\left(u_{1}, v_{1}\right)$ and $(u, v)$, and the fact that $K$ is two-connected to complete the path from $v_{1}$ to $v$.

To complete the proof, suppose that $a$ is $H$ and $b$ is in $K$. By Lemma 1 , since $H$ is two-vertex connected, there is a cycle in $H$ containing the vertex $a$ and the edge $\left(u_{1}, u\right)$. Therefore, there are vertex disjoint paths from $a$ to $u_{1}$ and $a$ to $u$ in $H$. Similarly, there are vertex disjoint paths from $b$ to $v_{1}$ and $b$ to $v$ in $K$. These together with the edges $\left(u_{1}, v_{1}\right)$ and $(u, v)$ provide the desired cycle. This completes the proof of Theorem 3 .

For any connected graph $G$ we define the canonical distance function $d(u, v)$ to be equal to the number of edges in a shortest path from $u$ to $v$. Note that $d$ is trivially a symmetric, nonnegative distance function satisfying the triangle inequality.

Theorem 4. Let $G=(V, E)$ be a graph which satisfies the following conditions:

(i) $G$ is two-connected.

(ii) Every vertex of $G$ has degree 2 or 3 .

(iii) $G$ is edge minimal, i.e., deleting any edge leaves a bridge.

(iv) Deleting any pair of edges in $G$ leaves a bridge in one of the resultant connected components.

Then $G$ is the unique minimum-cost two-connected network spanning Vfor the canonical distance function.

Proof. Let $X$ be the set of incidence vectors of edge sets of two-connected spanning networks on $V$. Let $P$ be the convex hull of $X$. Then, for any vector $c$ of distances, finding a minimum-cost two-connected spanning network of $V$ is the same as minimizing $c x$ over $P$. All members of $P$ satisfy the following inequalities:

$$
\begin{aligned}
& 0 \leqslant x(u, v) \leqslant 1 \text { for all } u, v \in V \\
& \sum(x(u, v): u \in S, v \in V \backslash S) \geqslant 2 \text { for all } \emptyset \neq S \subset V .
\end{aligned}
$$

Note that here, as throughout this paper, $(u, v)$ denotes the edge joining vertices $u$ and $v$ and $(v, u)$ is just another way of referring to the same edge. This also applies in the above: $x(u, v)$ and $x(v, u)$ are the same variable.

Let $\hat{x}$ be the incidence vector of $E$. We show that $G$ is the unique minimum cost two-connected network spanning $V$ by showing that $\hat{x}$ is the unique integer member of $P$ that minimizes $d x$ over $P$. This we do by considering the relaxation of the convex hull of $X$ obtained by only taking the constraints (1) and (2), and showing 
that $\hat{x}$ is the unique integer optimum solution to the linear program

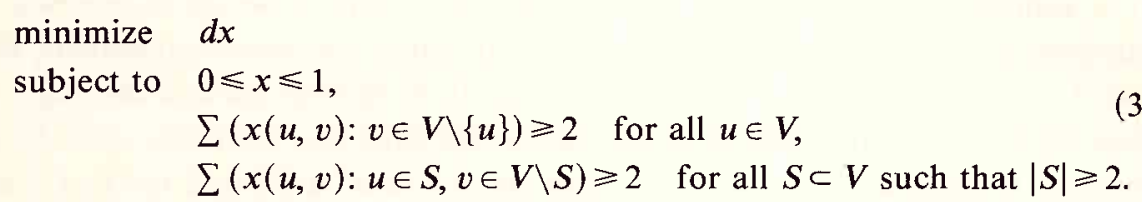

The dual linear program is

$$
\begin{array}{ll}
\operatorname{maximize} & \sum_{u \in V} 2 r_{u}+\sum\left(2 w_{S}: S \subset V,|S| \geqslant 2\right)-\sum_{u, v \in V} p(u, v) \\
\text { subject to } & r, w, p \geqslant 0, \\
& r_{u}+r_{v}+w(u, v)-p(u, v) \leqslant d(u, v) \quad \text { for all } u, v \in V,
\end{array}
$$

where for any $u, v \in V$ we let $w(u, v)=\sum\left(w_{S}: S \subset V,|S| \geqslant 2\right.$ and $\left.|S \cap\{u, v\}|=1\right)$.

We prove that $\hat{x}$ is the unique optimum solution to (3) by constructing a feasible solution to the dual which also satisfies the complementary slackness conditions for optimality and then showing that it is, in fact, the unique integer solution to (3) which satisfies these conditions. These complementary slackness conditions are:

$$
\begin{aligned}
& x(u, v)>0 \text { implies } r_{u}+r_{v}+w(u, v)-p(u, v)=d(u, v), \\
& r_{u}>0 \text { implies } \sum(x(u, v): v \in V \backslash\{u\})=2, \\
& w_{S}>0 \text { implies } \sum(x(u, v): u \in S, v \in V \backslash S)=2, \\
& p(u, v)>0 \text { implies } x(u, v)=1 .
\end{aligned}
$$

If $G=(V, E)$ is a single cycle then trivially $G$ is the unique two-connected spanning network on $V$ of minimum cost (equal to $|E|$ ). So assume that $G$ contains some vertices of degree three. Let $\bar{G}$ be the graph obtained from $G$ by replacing each maximal path of degree two vertices in $G$ with an edge. Then $\bar{G}$ is a cubic graph, although not necessarily simple, such that $G$ is a subdivision of $\bar{G}$. The vertices of $\bar{G}$ are the degree three vertices of $G$. Each edge $(u, v)$ of $\bar{G}$ corresponds to a path $u,\left(u, w_{1}\right), w_{1},\left(w_{1}, w_{2}\right), \ldots,\left(w_{k}, v\right), v$ in $G$ where $w_{1}, w_{2}, \ldots, w_{k}$ are degree two vertices of $G$. Since $G$ was two-connected, so too must be $\bar{G}$. Let $C$ be the set of edges of $\bar{G}$ which belong to two-edge cutsets, and let $P^{0}$ be the set of corresponding paths of $G$. Let $P^{1}$ be the set of paths of $G$ corresponding to edges of $\bar{G}$ not in $C$.

The following result is well known: The set $C$ partitions into $C_{1} \cup C_{2} \cup \cdots \cup C_{m}$ such that $\{j, k\}$ is a two-edge cutset in $\bar{G}$ if and only if $\{j, k\} \subseteq C_{i}$ for some $i$. (This follows easily from the fact that if $\{j, k\}$ and $\{k, 1\}$ are two-edge cutsets, then it we delete just $k$, both $j$ and 1 become bridges. Therefore, if we delete $1, j$ and add back $k$, the resulting graph must be disconnected, i.e., $\{j, 1\}$ is also a two-edge cutset. Therefore, "form a two-edge cutset" is an equivalence relation and the $C_{i}$ are the equivalence classes.)

Let $i \in\{1,2, \ldots, m\}$ and consider the graph $\bar{G} \backslash C_{i}$. It consists of at least two components, each has exactly two edges of $\bar{G}$ in its coboundary, and moreover, 
each such component must be two-connected. Now we define

$$
\begin{aligned}
& \bar{r}_{v}= \begin{cases}1 & \text { if } v \text { is a degree } 2 \text { vertex in a path of } P^{1}, \\
\frac{1}{2} & \text { if } v \text { is a degree } 2 \text { vertex in a path of } P^{0}, \\
0 & \text { if } v \text { is a degree } 3 \text { vertex; }\end{cases} \\
& \bar{p}(u, v)= \begin{cases}1 & \text { if } u, v \text { are adjacent degree } 2 \text { vertices in a path of } P^{1}, \\
0 & \text { otherwise; }\end{cases} \\
& \bar{w}_{S}=\left\{\begin{array}{cc}
\frac{1}{2} & \text { if } S \text { is the vertex set of a connected component formed } \\
\text { by choosing one } C_{i} \\
\text { and deleting all paths in } P^{0} \text { corresponding to edges of } C_{i}, \\
0 & \text { otherwise. }
\end{array}\right.
\end{aligned}
$$

Note that $\bar{w}$ contributes to (4) for an edge $j$ of $G$ if and only if $j$ is an end edge of a path of $P^{0}$.

We must verify that $\bar{r}, \bar{p}, \bar{w}$ is feasible and that it satisfies complementary slackness with respect to $\hat{x}$, the incidence vector of $E$. We have immediately that (6)-(8) are satisfied. We now verify that (4) and (5) hold. In doing so we will also determine which pairs $u, v$ of vertices satisfy $r_{u}+r_{v}+w(u, v)-p(u, v)=d(u, v)$. It is straightforward to verify that this holds for all edges $(u, v) \in E$.

For any $S \subseteq V$ define $\delta(S)=\{(u, v) \in E: u \in S, v \in V \backslash S\}$. That is, $\delta(S)$ is the coboundary of $S$. We let $\Delta(S)=\left\{p \in P^{0} \cup P^{1}\right.$ : one end of $p$ is in $S$ and the other end is in $V \backslash S\}$.

Let $u, v \in V$ be nonadjacent in $G$ and let $\pi$ be a shortest path in $G$ from $u$ to $v$. Then $d(u, v)=|E(\pi)|$. This path consists of a sequence $p_{0}, p_{1}, \ldots, p_{k}$ where, for each $i \in\{1,2, \ldots, k-1\}, p_{i}$ is a path of $P^{0} \cup P^{1}$ and $p_{0}$ and $p_{k}$ are partial or complete paths from $P^{0} \cup P^{1}$. (We may even have $k=0$, i.e., $p_{0}=p_{k}$.) Since $(u, v) \notin E, \bar{p}(u, v)=$ 0 . Let $S \subseteq V$ satisfy $u \in S, v \in V \backslash S$. Then an odd number of edges of $\pi$ must belong to $\delta(S)$ and, if $\bar{w}_{S}>0$, then $|\delta(S)|=2$. Therefore:

(A) For any $S \subseteq V$ such that $\bar{w}_{S}>0, u \in S$ and $v \in V \backslash S$, exactly one edge of $\pi$ belongs to $\delta(S)$. Therefore, for any such $S$ there is a unique $i \in\{0,1,2, \ldots, k\}$ such that $p_{i} \in \Delta(S)$. Therefore,

$$
|E(\pi)|=\sum_{i=0}^{k}\left|E\left(p_{i}\right)\right| \geqslant \sum\left(\left|E\left(p_{i}\right)\right|: i=0,1, \ldots, k ; p_{i} \in P^{0}\right) \geqslant \bar{w}(u, v) .
$$

(B)(1) Suppose that $u$ and $v$ are of degree three. Then $\bar{r}_{u}=\bar{r}_{v}=0$ so (4) holds and we have equality in (4) if and only if each path $p_{i}$ consists of a single edge and each $p_{i} \in P^{0}$.

(2) Suppose that $u$ is of degree 3 and $v$ is of degree 2. Then again (4) holds and we have equality if and only if, for each $i \in\{0,1, \ldots, k-1\}, p_{i} \in P^{0} ;\left|E\left(p_{i}\right)\right|=1$ and $v$ is a degree 2 vertex of a path of $P^{0}$ or $P^{1}$ which is adjacent in $G$ to the degree 3 vertex of $G$ which is common to $p_{k-1}$ and $p_{k}$. 
(3) Finally, if both $u$ and $v$ are of degree two, then, again (4) holds and we have equality if and only if for $i \in\{1,2, \ldots, k-1\}, p_{i} \in P^{0}$ and $\left|E\left(p_{i}\right)\right|=1$, and each of $p_{0}$ and $p_{k}$ consists of a single edge.

One additional consequence of (A) which we use is the following.

(C) Suppose $(u, v)$ satisfies (4) with equality but $(u, v) \notin E$. Suppose, in addition, that there is some part $C_{i}$ of the cutset partition of $\bar{G}$ such that, when we delete the paths of $P^{0}$ corresponding to the edges of $C_{i}$ from $G, u$ and $v$ are in distinct components, say $K_{1}$ and $K_{2}$, of the resulting graph. Then $K_{1}$ and $K_{2}$ must be joined by a path of $P^{0}$ which consists of a single edge.

Since we have shown that $\bar{r}, \bar{p}, \bar{w}$ is feasible and since (4) holds with equality for all edges of $E$, we have established that $G$ is a minimum-cost two-connected spanning network on $V$, and also that $\bar{r}, \bar{p}, \bar{w}$ is an optimal dual solution. Therefore, we can use complementary slackness to deduce properties which must be satisfied by any optimal integer-valued solution $x$ to (3).

By (7):

(D) Suppose we choose any $C_{i}$ from the cutset partition of $C$ and delete from $G$ those paths in $P^{0}$ which correspond to edges of $C_{i}$. Let $K$ be the vertex set of a component of the resulting graph. Then $\sum_{u \in K, v \in V \backslash K} x(u, v)=2$.

By (6):

(E) For any degree two vertex $u$ of $G$, we must have $\sum_{v \in V \backslash\{u\}} x(u, v)=2$.

Also, we have:

(F) For any edge $(u, v) \in E$ where $u, v$ have degree two in $G$, we must have $x(u, v)=1$.

For if $(u, v)$ belongs to a path of $P^{1}$ then this is just (8). Suppose $(u, v)$ belongs to a path $\pi$ of $P^{0}$. If there exists another vertex $w$ adjacent in $G$ to $v$ such that $w$ has degree two, then $(u, v)$ and $(v, w)$ are the only pairs including $v$ which satisfy (4) with equality, so (F) must hold. So suppose $\pi$ contains exactly three edges and $x(u, v)=0$. Let $C_{i}$ be the member of the cutset partition containing the edge corresponding to $\pi$. Deleting the paths corresponding to edges of $C_{i}$ from $G$ we obtain at least two components. Let $K_{1}$ and $K_{2}$ be the components containing the ends of $\pi$ and assume that $u$ is adjacent in $G$ to a vertex of $K_{1}$. By (B), the only vertices $w \neq v$ for which $(u, w)$ satisfies (4) with equality belong to $K_{1}$. Therefore, by (D) and (E), $u$ is the only vertex of $G$ adjacent to a node $w$ of $K_{1}$ such that $x(u, w)=1$. But this means that the support of $x$ is not connected, contradictory to $x$ being a feasible solution to (3).

Let $E^{=}$be the set of edges of the complete graph on vertex set $V$ which satisfy (4) with equality. As we have noted, $E \subseteq E^{-}$. Let $G^{\prime}=\left(V, E^{\prime}\right)$ be any minimum-cost two-connected spanning subgraph. Then $E^{\prime} \subseteq E^{=}$. We show that $G^{\prime}=G$ by considering two cases. Recall that $m$ is the number of parts of the partition $C_{1} \cup C_{2} \cup \cdots \cup C_{m}$ of $C$.

Case 1. $m=0$. Then $\bar{G}$ is three-edge-connected and so each edge of $\bar{G}$ is subdivided at least twice in $G$, for otherwise we would contradict (iii) or (iv). By (E), every degree two vertex of $G$ also has degree two in $G^{\prime}$. Moreover, by (F), any 
edge of $G$ which joins two vertices of degree two (in $G$ ) is present in $G^{\prime}$. Suppose $(u, v) \in E^{-} \backslash E$ and $(u, v) \in E^{\prime}$. Both $u$ and $v$ must be of degree two in $G$, and hence in $G^{\prime}$. Since $(u, v) \notin E, d(u, v) \geqslant 2$. But since $d(u, v)=\bar{r}_{u}+\bar{r}_{v} \leqslant 2$, we must have $d(u, v)=2$. Let $s$ be adjacent in $G$ to $u$ and $v$. If $s$ is of degree two, then by (F), each of $(s, u)$ and $(s, v)$ must be in $E^{\prime}$. By (E), $\{u, v, s\}$ must therefore be adjacent only to each other in $G^{\prime}$, contradicting $G^{\prime}$ being two-connected. Therefore $s$ is of degree 3. For any $w \in V \backslash\{s\}, \bar{r}_{w}+\bar{r}_{s}=\bar{r}_{w}+0 \leqslant 1$. Therefore $(s, w) \in E^{=}$if and only if $w$ is a degree two vertex of $G$ adjacent to $s$. In other words, the neighbours of $s$ in $G^{\prime}$ must be a subset of at least two of the neighbours of $s$ in $G$. Let $w$ be the vertex, other than $u$ and $v$, adjacent to $s$. By (F), each of $u$ and $v$ is adjacent to other degree two vertices of $G^{\prime}$ as well as each other, so, by (E), $w$ is the only vertex of $G^{\prime}$ which can be adjacent to $s$, contradictory to $G^{\prime}$ being two-connected. Therefore $E^{\prime}=E$ and so $G$ is the unique optimum.

Case 2. $m>0$. We proceed by induction on the size of $G$. For each $i \in\{1,2, \ldots, m\}$, it is easy to see that each $C_{h}$, for $h \in\{1,2, \ldots, m\} \backslash\{i\}$, must be contained in the edge set of one component of $\bar{G} \backslash C_{i}$. Thus we are able to choose $i \in\{1,2, \ldots, m\}$ and a component $\bar{K}$ of $\bar{G} \backslash C_{i}$ in such a way that $\bar{K}$ contains no edge of $C$. Let $\bar{C}_{i}$ be the set of edges of $G$ belonging to those paths of $P^{0}$ which correspond to edges of $C_{i}$. Let $\hat{G}$ be obtained from $G$ by deleting the edges of $\bar{C}_{i}$. Then components of $\hat{G}$ can be of two types: some will correspond to components of $\bar{G} \backslash C_{i}$ and others will consist of isolated degree two vertices of $G$. We call the latter trivial and the former nontrivial. Note that by (iv), $\left|\bar{C}_{i}\right| \geqslant 3$. Therefore $\hat{G}$ has at least three components, at least two of which are non-trivial, and the edges of $\bar{C}_{i}$ join these components in a cyclic fashion. Let $K$ be the component corresponding to $\bar{K}$. See Fig. 3.

Now let $(u, v)$ be any edge of $E^{-} \backslash E$ such that $u$ and $v$ belong to different components of $\hat{G}$. We show that an edge of $G$ joins these components. Only edges of $E$ join vertices belonging to trivial components, so at least one of $u$ and $v$ belongs to a nontrivial component. If both $u$ and $v$ belong to nontrivial components, then by $(C)$ these components are joined by an edge of $G$. If one is in a trivial component and the other in a nontrivial component then again these components are joined by an edge of $G$.

By (D) and (E) each component of $\hat{G}$ must have exactly two edges of $E^{\prime}$ in its coboundary. Let $\tilde{G}$ and $\tilde{G}^{\prime}$ be obtained from $G$ and $G^{\prime}$ respectively by shrinking the vertex sets of all components of $\hat{G}$ other than $K$. It follows that $\tilde{G}$ and $\tilde{G}^{\prime}$ must agree on all edges except possibly those incident with a vertex of $K$. We now show that

$$
\tilde{G}=\tilde{G}^{\prime}
$$

Let $v_{1}$ and $v_{2}$ be the vertices of $K$ adjacent in $G$ to vertices of other components of $\hat{G}$. Let $u_{1}$ and $u_{2}$ be the vertices of $K$ adjacent in $G^{\prime}$ to vertices of other components of $\hat{G}$. Then $u_{1}$ and $u_{2}$ must be distinct and each must be either equal to $v_{1}$ or $v_{2}$ or else be a degree two vertex adjacent to $v_{1}$ or $v_{2}$ in $G$, since by (D) these are the 


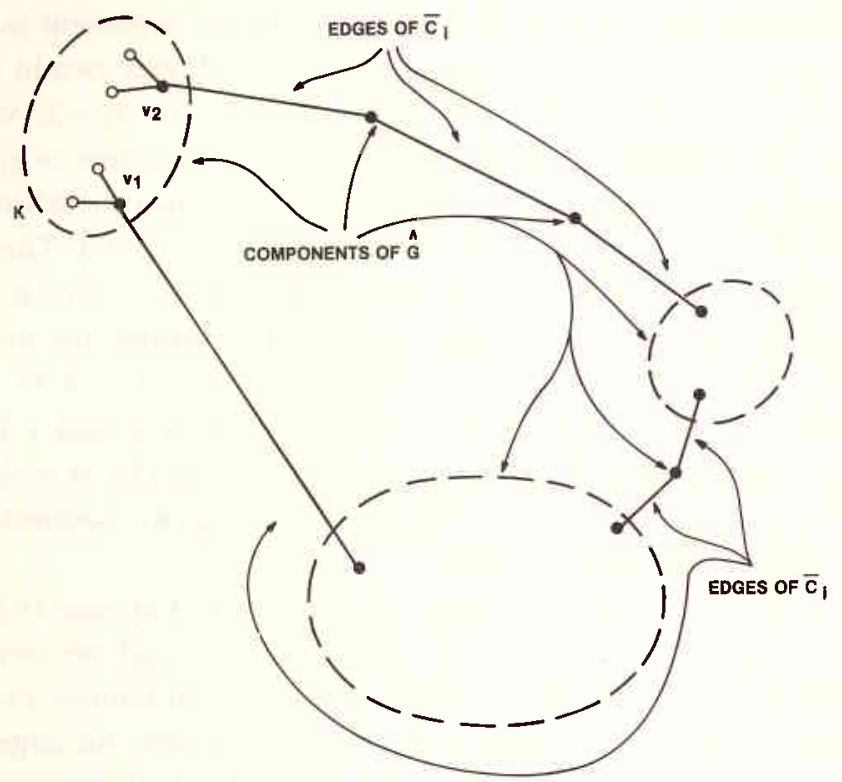

Fig. 3.

only vertices of $K$ joined by an edge of $E^{=}$to a vertex not in $K$. We show that $\left\{u_{1}, u_{2}\right\}=\left\{v_{1}, v_{2}\right\}$. Suppose that this is not the case and that $u_{1}$ is of degree two and is adjacent to $v_{1}$. Since $K$ contains no edges belonging to paths of $P^{0}, u_{1}$ is joined by an edge $\left(u_{1}, \hat{w}\right)$ of $K$ to another degree two vertex $\hat{w}$ of $K$. By $(\mathrm{F}),\left(u_{1}, \hat{w}\right) \in E^{\prime}$ and by (E), $u_{1}$ has degree two in $G^{\prime}$. Let $w$ be the vertex of $G^{\prime}$ other than $\hat{w}$ adjacent in $G^{\prime}$ to $u_{1}$. Then the only vertices to which $v_{1}$ can be adjacent in $G^{\prime}$ are the other incident vertex $\bar{u}_{1}$ of $K$ plus vertices in other components of $\hat{G}$. See Fig. 4.

Since $v_{1}$ has degree at least two in $G^{\prime}, v_{1}$ must be adjacent in $G^{\prime}$ to a vertex $\bar{w}$ which by (B) must lie in the component $\bar{K}$ of $\hat{G}$ which contains $w$. But by (D), $\left(u_{1}, w\right)$ and $\left(v_{1}, \bar{w}\right)$ are the only edges of the coboundary of $K$ and $\bar{K}$ in $G^{\prime}$. Therefore $V(K) \cup V(\bar{K})$ induces a connected component of $G^{\prime}$. But since $\hat{G}$ has

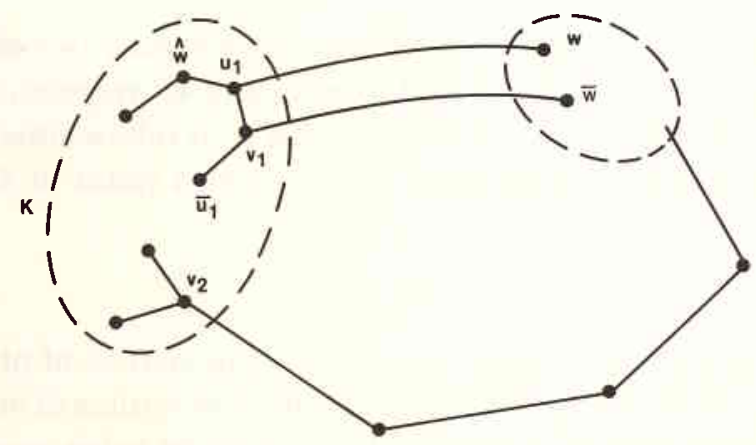

Fig. 4. 
at least three components, this is a contradiction. Therefore $v_{1}$ and $v_{2}$ are the two vertices of $K$ adjacent in $G^{\prime}$ to vertices not in $K$.

Let $\tilde{d}$ be the set of distances defined by $\tilde{G}$. Since $\tilde{G}$ satisfies (i)-(iv), by induction, $\tilde{G}$ is the unique minimum-cost two-connected spanning subgraph on this vertex set. Suppose $\tilde{G}^{\prime} \neq \tilde{G}$. We have seen that they coincide on all edges not in $K$, so this means that $\tilde{d}\left(E^{\prime}(K)\right)>\tilde{d}(E(K))=d(E(K))$. But $d\left(E^{\prime}(K)\right) \geqslant \tilde{d}\left(E^{\prime}(K)\right)$, since the distance between each pair of vertices is at least as large in $G$ as in $\tilde{G}$. Therefore $d\left(E^{\prime}(K)\right)>d(E(K))$. Therefore we can replace $G^{\prime}[V(K)]$ with $K$ in $G^{\prime}$ and obtain a two-connected spanning subgraph of $V$ with lower cost, with respect to $d$, than that of $G^{\prime}$, a contradiction. Hence (9) is established.

Now let $\tilde{H}$ and $\tilde{H}^{\prime}$ be obtained from $G$ and $G^{\prime}$ respectively by shrinking the vertex set of the component $K$. Let $\bar{d}$ be the vector of distances in $\tilde{H}$. Since $\tilde{H}$ satisfies (i)-(iv), by induction $\tilde{H}$ is the unique minimum-cost two-connected spanning subgraph of the vertex set of $\tilde{H}$, with distance $\bar{d}$. Now $\bar{d}(E(\tilde{H}))=d(E)-|E(K)|$. Since $G$ and $G^{\prime}$ were both minimum cost with respect to $d$, we had $d(E)=d\left(E^{\prime}\right)$. Since $\bar{d}(u, v) \leqslant d(u, v)$ for all $(u, v)$, and using $(9), d\left(E\left(\tilde{H}^{\prime}\right)\right) \leqslant d(E)-|E(K)|=$ $\bar{d}(E(\tilde{H}))$. There $\tilde{H}^{\prime}$ is also a minimum-cost two-connected spanning subgraph, with respect to $\bar{d}$, so $\tilde{H}=\tilde{H}^{\prime}$. But this implies that $G=G^{\prime}$, as required, completing the proof of Case 2.

Theorems 3 and 4 are useful for studying optimal two-connected solutions because we may restrict our attention to cycles, cubic two-connected graphs with subdivided edges, and graphs of the form shown in Fig. 5. We note that contracting the degree two vertices in the graph of Fig. 5 results in two vertices with three parallel edges between them; we treat this as a separate case since normally such a graph is not considered to be two-vertex connected. Such graphs, however, form an important class of two-connected solutions. They are the unique minimal two-connected graphs which are not cycles.

Corollary 5. Any two-connected graph $G=(V, E)$ satisfying conditions (a) and (b) of Theorem 3, and which is not a cycle, contains the graph shown in Fig. 5 as a vertex-induced subgraph.

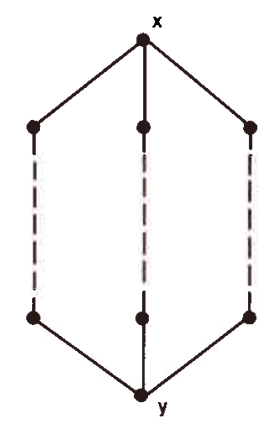

Fig. 5. Graph in which the broken rules are paths consisting of one or more edges. 
Proof. Since $G$ is two connected, it contains a cycle $C$. By condition (b) of Theorem $3, C$ contains at least three vertices. Since $G$ is not itself a cycle, there must be at least one vertex $z$ not in $C$. By Lemma $1, z$ is contained on a common cycle with every vertex in $C$. By condition (a) of Theorem 3 , this implies that there exist two vertices $x$ and $y$ in $C$ with vertex paths to $z$ which avoid $C$ (except for the end nodes $x$ and $y$ ). Condition (b) of Theorem 3 implies that this vertex-induced subgraph must be of the form shown in Fig. 5 .

Another consequence of Theorem 3 is that an optimal two-connected network need not be planar since $K_{3.3}$ with all edges subdivided two or more times satisfies conditions (a) and (b). This contrasts with the case of points in the plane where if the Euclidean metric is used, then there always exists a planar optimal solution. (Clearly, condition (a) precludes the possibility that a subgraph will be homeomorphic to $K_{5}$.)

\section{Comparison to traveling salesman problem}

In this section we discuss the relationship of the minimum weight two-connected spanning network problem to the traveling salesman problem $[1,5,24,30]$. Much work has been done on the worst-case analysis of heuristics to obtain minimumweight cycles $[4,8,15-17,29,32]$. By Theorem 4 , we know that a cycle need not produce an optimal two-connected solution. We show that Theorem 3 makes it easy to establish a tight worst-case bound on the ratio of an optimal cycle length to an optimal two-connected length. We also relate these results to a 'Steiner' version of the problem.

Before proceeding, we need a few additional definitions and a useful lemma. A perfect matching of $W \subseteq V$ in a graph $G=(V, E)$ is a subset of edges $M \subseteq E$ which meet every vertex in $W$ exactly once. For any set of vertices $V$ and distance function $d(\cdot)$, we let $C_{\text {opt }}(V)$ denote an optimal cycle and $\mathrm{TC}_{\text {opt }}(V)$ denote an optimal two-connected solution of weight $d\left(C_{\text {opt }}(V)\right)$ and $d\left(\mathrm{TC}_{\text {opt }}(V)\right)$, respectively. When the context is clear, we drop the specification of $V$.

The following result follows easily from Edmonds' characterization of the matching polytope [12].

Lemma 6 [28]. Let $G=(V, E)$ be a cubic, two-edge connected graph. Then for any nonnegative edge weight function $c(\cdot)$, there is a perfect matching $M$ such that $c(M) \leqslant \frac{1}{3} c(E)$.

In our search for a worst-case example for the ratio $d\left(C_{\text {opt }}\right) / d\left(\mathrm{TC}_{\text {opt }}\right)$, we may restrict our attention in the following useful ways. Suppose that $G=(V, E)$ is an optimal two-connected solution for the distance function $d(\cdot)$. By the triangle inequality, $d(u, v)$ is at most the length of a shortest path from $u$ to $v$ in $G$. Since we are looking for a worst-case example, we may make $d(u, v)$ equal to this length 
for all $(u, v) \notin E$. Furthermore, under the reasonable assumption that $d(\cdot)$ is a rational-valued function, we may multiply all distances by a large enough number to obtain an integer-valued function. Finally, it is easy to see that any edge with integer weight $h>1$ can be subdivided into a path of length $h$ with all edges having weight one without increasing the cost of the solution or changing any of the other edges shortest-path lengths; furthermore, the best cycle in this enlarged problem cannot be shorter than the best cycle in the original problem. Therefore the worst case ratio will be realized by some graph with the canonical distance function.

Theorem 7. For any set of vertices $V$ and distance function $d(\cdot)$,

$$
d\left(C_{\mathrm{opt}}(V)\right) / d\left(\mathrm{TC}_{\mathrm{opt}}(V)\right) \leqslant \frac{4}{3} .
$$

Furthermore, this bound can be approached arbitrarily closely by the class of graphs shown in Fig. 5 with $d(\cdot)$ their canonical distance function.

Proof. Let $G=(V, E)$ be a minimum-weight two-connected spanning network satisfying the conditions of Theorem 3. Assume, without loss of generality, that $G$ is not a cycle, and that $d(\cdot)$ is the canonical distance function for $G$.

Let $\bar{V}$ be the set of degree three vertices of $G$. Then $G$ is a subdivision of the cubic bridgeless graph $\bar{G}=(\bar{V}, \bar{E})$ obtained by repeatedly contracting all edges adjacent to degree two vertices, and each $(u, v) \in \bar{E}$ corresponds to a maximal path in $G$, all of whose internal vertices have degree two. For each such $(u, v) \in \bar{E}$, let $\bar{d}(u, v)$ be the number of edges in the corresponding path. Then $\bar{d}(\bar{E})=d(E)$. By Lemma $6, \bar{G}$ has a perfect matching $M$ of weight at most $\frac{1}{3} \bar{d}(\bar{E})$. By duplicating the edges of the paths corresponding to the edges of $M$ in $G$, we obtain an Eulerian multigraph $G^{\prime}=\left(V, E^{\prime}\right)$ on $V$ and $d\left(E^{\prime}\right) \leqslant \frac{4}{3} d(E)$. Since $d$ satisfies the triangle inequality, we can apply a standard shortcutting argument to construct a hamiltonian circuit $C$ on $V$ such that $d(C) \leqslant d\left(E^{\prime}\right) \leqslant \frac{4}{3} d\left(\mathrm{TC}_{\mathrm{opt}}(V)\right)$.

We can see that the bound of $\frac{4}{3}$ is tight by considering the graph of Fig. 5 in which each path between the degree three vertices, call them $s$ and $t$, has $k$ interval vertices, the best cycle has distance $4 k+2$ under the canonical distance function. Since the graph has $3 k+3$ edges, this gives a ratio of $(4 k+2) /(3 k+3)$ which approaches $\frac{4}{3}$ as $k$ approaches $\infty$.

Theorem 7 was proved by Fredrickson and Ja'Ja' [15] for the case that the optimal solution to the two-connected problem is planar, and indeed, they observed that Lemma 6, if it were true, would imply the result for general solutions. (Ironically, [28] containing Lemma 6 appeared the year before [15].) Moreover, they conjectured a result which would imply Lemma 6 . This result is an easy consequence of Edmonds' matching polyhedron characterization [12] and was the tool used in [28] to prove Lemma 6 . The proof of Theorem 7 which we give here follows similar lines to that of [15]. However, the existence of Theorem 3, in particular, the fact that there always exists an optimum solution of maximum degree three, simplifies things. In [15] an arbitrary two-connected graph $G$ is first transformed into a cubic bridgeless 
graph $G^{\prime}$ by replacing high degree nodes with cycles and replacing degree two nodes with suitable subgraphs. All new edges are given cost zero. If $G$ is planar, so too will be $G^{\prime}$, so the Four Color Theorem can be used to show that the edges can be partitioned into three perfect matchings, at least one of which has cost at most $\frac{1}{3} d(G)$. A variation of the argument given above then constructs the desired cycle in $G$.

We note that any lower bound $\gamma$ for the worst-case ratio of a heuristic $H$ to an optimal cycle $C_{\text {opt }}$ produces a worst-case lower bound for an optimal two-connected solution $\mathrm{TC}_{\mathrm{opt}}$ of at least $\gamma$ since $d\left(\mathrm{TC}_{\mathrm{opt}}\right) \leqslant d\left(C_{\mathrm{opt}}\right)$. An upper bound of $\beta$ for the worst-case ratio of a heuristic $H$ to an optimal cycle $C_{\text {opt }}$ produces a worst-case upper bound of at most $\frac{4}{3} \beta$ using Theorem 7 .

The following interesting lower bound on $d\left(\mathrm{TC}_{\mathrm{opt}}\right)$, which relates closely to the proof technique of Theorem 4, was obtained by W.H. Cunningham. The subtour polytope of the traveling salesman problem is the set of all solutions to the following linear system:

$$
\begin{aligned}
& 0 \leqslant x \leqslant 1, \\
& \sum(x(u, v): v \in V \backslash\{u\})=2 \text { for all } u \in V, \\
& \sum(x(u, v): u \in S, v \in V \backslash S) \geqslant 2 \text { for all } S \subset V \text { such that }|S| \geqslant 2 .
\end{aligned}
$$

Note that this differs from (3) only in that the inequalities corresponding to $u \in V$ have been made into equations, which are satisfied by the incidence vector of any Hamiltonian cycle, but not by the incidence vector of an arbitrary two-connected spanning subgraph. Let $S_{\text {opt }}$ be a solution to the subtour polytype for which $d x$ is minimized, and let $d\left(S_{\text {opt }}\right)$ denote its value. Trivially $d\left(S_{\text {opt }}\right) \leqslant d\left(C_{\text {opt }}\right)$, but in fact it also provides a lower bound on $d\left(\mathrm{TC}_{\mathrm{opt}}\right)$.

Theorem 8 (W.H. Cunningham). For any set $V$ of vertices and distance function $d(\cdot)$, $d\left(S_{\mathrm{opt}}\right) \leqslant d\left(\mathrm{TC}_{\mathrm{opt}}\right)$.

Proof. Let $H$ be a minimum-cost two-connected spanning network. Doubling each edge in $H$ yields a multigraph $H^{\prime}$ spanning $V$ and having cost twice $d\left(\mathrm{TC}_{\text {opt }}\right)$. Each vertex $v$ has even degree in $H^{\prime}$ and $H^{\prime}$ is four-edge connected. Successively choose a vertex $v$ of degree more than four and a pair $(u, v),(v, w)$ of incident edges such that deleting these edges and adding the edge $(u, w)$ preserves the four-edge connectivity of $H^{\prime}$. (This is always possible by a result of Lovász [25].) Notice that this step also preserves the even degree property of $H^{\prime}$ and by the triangle inequality, the cost of the $H^{\prime}$ does not increase. When this step can no longer be performed, $H^{\prime}$ is a four regular, four-edge-connected, spanning network of $V$ of cost at most twice $d\left(\mathrm{TC}_{\mathrm{opt}}\right)$. Define $\bar{x}(u, v)$ to be $\frac{1}{2}$ the number of edges of $H^{\prime}$ joining $u$ and $v$. Then $\bar{x}$ belongs to the subtour polytope, and $d\left(S_{\text {opt }}\right) \leqslant d \bar{x} \leqslant d\left(\mathrm{TC}_{\mathrm{opt}}\right)$ as required.

An interesting variation of the minimum-weight two-connected spanning network problem is to specify a subset of special vertices $D \subseteq V$ and find a minimum-weight 
two-connected network spanning $D$. (Other vertices may be used if they help reduce the overall cost.) We call this problem the Steiner two-connected network problem. Theorem 3 implies that any vertex in $V \backslash D$ used in an optimal network is of degree 3.

We note that the minimum Steiner tree problem is a well-studied [11, 13, 19, 20, 31] generalization of the minimum spanning tree problem. The next theorem provides a worst-case bound for the ratio of an optimal two-connected solution spanning $D$ and a Steiner two-connected solution. We denote an optimal Steiner two-connected solution by $\operatorname{STC}_{\text {opt }}(D, V)$ with weight $d\left(\operatorname{STC}_{\text {opt }}(D, V)\right)$. We omit $D$ and $V$ when the context is clear.

Theorem 9. For any set of vertices $V, D \subseteq V$ and distance function $d(\cdot)$,

$$
d\left(\operatorname{TC}_{\mathrm{opt}}(D)\right) / d\left(\operatorname{STC}_{\mathrm{opt}}(D, V)\right) \leqslant \frac{4}{3} \text {. }
$$

Proof. Let $G=(W, E)$ be an optimal Steiner two-connected solution; clearly, $D \subseteq$ $W$. Now, $\operatorname{TC}_{\text {opt }}(D) \leqslant C_{\text {opt }}(D) \leqslant C_{\text {opt }}(W)$ and $\operatorname{STS}_{\text {opt }}(D, V)=\operatorname{STC}_{\text {opt }}(D, W)=$ $\mathrm{TC}_{\text {opt }}(W)$ imply that

$$
\operatorname{TC}_{\text {opt }}(D) / \operatorname{STC}_{\text {opt }}(D, V) \leqslant C_{\text {opt }}(W) / \mathrm{TC}_{\text {opt }}(W) \leqslant \frac{4}{3} .
$$

The final inequality follows from Theorem 7 .

We note that the Steiner two-connected network problem is NP-hard even for the special distance functions considered in this paper. However, this problem can be solved efficiently for special classes of graphs even for arbitrary distance functions; e.g., for outerplanar graphs [36]. It is easy to see that this problem can be efficiently solved for the more general series-parallel graphs as well, in a manner similar to those used to solve other problems on these graphs [27, 35]. A different generalization of outerplanar graphs, called D-planar graphs, requires that the graph have a planar representation where all vertices in $D$ be on a common face. The Steiner tree problem $[13,31]$ and the more general concave-cost network flow problem [13] can be efficiently solved on $D$-planar graphs with arbitrary weight functions. It can be shown that an optimal two-connected solution spanning $D$ will be a cycle passing through the vertices in $D$ in the order they appear around their common face in the planar embedding. We leave as an open question whether this fact can be used to derive an efficient solution procedure.

\section{Concluding remarks}

We have studied the problem of finding a minimum-weight two-connected spanning network given a non-negative, symmetric distance function satisfying the triangle inequality. Our results apply equally to two-vertex connected and two-edge connected requirements and are applicable to the design of survivable communication and transportation networks. 
Strong structural properties of optimal two-connected spanning networks were derived in Section 2. Recent work [3] extends Theorem 3 to minimum-weight $k$-connected solutions for all $k \geqslant 2$. That is, there exists a minimum-weight $k$-edge (resp. $k$-vertex) connected network satisfying (a) every vertex is degree $k$ or $k-1$, and (b) deleting any $1,2, \ldots$, or $k$ edges does not result in all connected components being $k$-edge (resp. $k$-vertex) connected. It is also shown that the analogue to Theorem 4 does not hold for $k \geqslant 3$.

An important research direction which has not yet received adequate attention is to determine valid inequalities for the polyhedron of the convex hull of $k$ connected networks. Obtaining 'nice' classes of inequalities would be useful in further understanding the problem and could be used in cutting-plane approaches to solving practical problems to optimality. This approach has been successfully applied to the traveling salesman problem [7, 9, 10]. A first step in this direction is taken in [21].

Finally, we note that the transformations implicit in the proof of Theorem 3 along with methods similar to those used for the traveling salesman problem have been implemented into an Interactive Network Design System (INDS) on the IBM PC [26]. These methods have been tested on real-world problems of designing survivable fiber optic communication networks and consistently produce 'near-optimal' networks.

\section{Acknowledgement}

We are grateful to the referees whose comments improved the presentation of this paper as well as to Bill Cunningham for giving us permission to include Theorem 8 .

\section{References}

[1] Bell Laboratories, "The traveling salesman problem: 1934-1978," Bibliography No. 367 (Holmdel, NY, 1979).

[2] C. Berge, Graphs and Hypergraphs (North-Holland, Amsterdam, 1973).

[3] D. Bienstock, E.F. Brickell and C.L. Monma, "Properties of $k$-connected networks," SIAM Journal on Discrete Mathematics, to appear.

[4] N. Christofides, "Worst-case analysis of a new heuristic for the traveling salesman problem," Technical Report, GSIA, Carnegie-Mellon University (Pittsburgh, PA, 1976).

[5] N. Christofides, "The traveling salesman problem," in: N. Christofides et al., eds., Combinatorial Optimization (Wiley, New York, 1979) pp. 131-150.

[6] N. Christofides and C.A. Whitlock, "Network synthesis with connectivity constraints-A survey," in: J.P. Brans, ed., Operational Research 81 (North-Holland, Amsterdam, 1981) pp. 705-723.

[7] G. Cornuéjols, J. Fonlupt and D. Naddef, "The traveling salesman problem on a graph and some related integer polyhedra," Mathematical Programming 33 (1985) 1-27.

[8] G. Cornuéjols and G.L. Nemhauser, "Tight bounds on Christofides' traveling salesman heuristic," Mathematical Programming 14 (1978) 116-121.

[9] H. Crowder and M.W. Padberg, "Solving large-scale symmetric traveling salesman problems to optimality," Management Science 26 (1980) 495-509. 
[10] G.B. Dantzig, D.R. Fulkerson and S.M. Johnson, "On a linear programming, combinatorial approach to the traveling salesman problem," Operations Research 7 (1959) 58-66.

[11] S.E. Dreyfus and R.A. Wagner, "The Steiner problem in graphs," Networks 1 (1971) 195-207.

[12] J. Edmonds, "Maximum matching and a polytope with 0-1 vertices," Journal of Research of the National Bureau of Standards 698 (1965) 125-130.

[13] R.E. Erickson, C.L. Monma and A.F. Veinott Jr., "Send-and-split method for minimum-concave-cost network flows," Mathematics of Operations Research 12 (1987) 634-664.

[14] K.P. Eswaran and R.E. Tarjan, “Augmentation problems," SIAM Journal on Computing 5 (1976) 653-665.

[15] G.N. Frederickson and J. Ja'Ja', "On the relationship between the biconnectivity augmentation and traveling salesman problem," Theoretical Computer Science 13 (1982) 189-201.

[16] A.M. Frieze, "Worst-case analysis of algorithms for traveling salesman problems," Operational Research Verfahren 32 (1979) 93-112.

[17] A.M. Frieze, G. Galbiati and F. Maffioli, "On the worst-case performance of some algorithms for the asymmetric traveling salesman problems," Networks 12 (1982) 23-39.

[18] M.R. Garey and D.S. Johnson, Computers and Intractability: A Guide to the Theory of NPCompleteness (Freeman, San Francisco, CA, 1979).

[19] E.N. Gilbert and H.O. Pollak, "Steiner minimal trees," SIAM Journal on Applied Mathematics 16 (1968) $1-29$

[20] R.L. Graham and P. Hell, "On the history of the minimum spanning tree problem," Annals of the History of Computing 7 (1985) 41-57.

[21] M. Grötschel and C.L. Monma, "Integer polyhedra arising from certain network design problems with connectivity constraints," SIAM Journal on Discrete Mathematics, to appear.

[22] M. Grötschel and M.W. Padberg, "On the symmetric traveling salesman problem I: Inequalities," Mathematical Programming 16 (1979) 265-280.

[23] M. Grötschel and M.W. Padberg, "On the symmetric traveling salesman problem II: Lifting theorems and facets," Mathematical Programming 16 (1979) 281-302.

[24] E.L. Lawler, J.K. Lenstra, A.H.G. Rinnooy Kan and D.B. Shmoys, eds., The Traveling Salesman Problem (Wiley, New York, 1985).

[25] L. Lovász, "On some connectivity properties of Eulerian graphs," Acta Mathematica Academiae Scientiarum Hungaricae 28 (1976) 129-138.

[26] C.L. Monma and D.F. Shallcross, "Methods for designing survivable communication networks," Operations Research (1989).

[27] C.L. Monma and J.B. Sidney, "Sequencing with series-parallel precedence constraints," Mathematics of Operations Research 4 (1979) 215-224

[28] D. Naddef and W.R. Pulleyblank, "Matching in regular graphs," Discrete Mathematics 34 (1981) 283-291.

[29] C. Papadimitriou and U.V. Vazirani, "On two geometric problems related to the traveling salesman problem," Journal of Algorithms 5 (1984) 231-246.

[30] R.G. Parker and R.L. Rardin, "The traveling salesman problem: An update of research," Naval Research Logistics Quarterly 30 (1983) 69-96.

[31] J.S. Provan, "Convexity and the Steiner tree problem," Networks 18 (1988) 55-72.

[32] W.R. Pulleyblank, "On minimizing setups in precedence constrained scheduling," Operations Research Technical Report 81185, University of Bonn (Bonn, 1981).

[33] D. J. Rosenkrantz, R.E. Stearns and P.M. Lewis II, "An analysis of several heuristics for the traveling salesman problem," SIAM Journal on Computing 6 (1977) 563-581.

[34] K. Steiglitz, P. Weiner and D.J. Kleitman, "The design of minimum cost survivable networks," IEEE Transactions on Circuit Theory 16 (1969) 455-460.

[35] K. Takamizawa, T. Nishizek and N. Saito, "Linear-time computability of combinational problems on series parallel graphs," Journal ACM 29 (1982) 632-641.

[36] J.A. Wald and C. Colbourn, "Steiner trees in outerplanar graphs," Congressus Numeratum 36 (1982) 15-22. 